\title{
Scientists need to better communicate the links between pandemics and global environmental change
}

To the Editor - The current COVID-19 pandemic is often imagined as a challenge humanity has to weather, with additional, different ones - including climate change lurking right behind. This conceptualization of the pandemic as a human health crisis, juxtaposed to other, separate issues affecting the planet misses the bigger picture: that this pandemic is part of a syndrome of human effects on the planet. The pandemic and global environmental change are intimately intertwined at multiple levels, and this must be more clearly articulated to the public and in policy.

In Fig. 1, we illustrate the interconnections of anthropogenic global change and the COVID-19 pandemic. The virus causing the COVID-19 pandemic (SARS-CoV-2) is a new pathogen to humans that has spread globally beyond its initial reach as a consequence of human movement; this spread can be viewed as bearing similarities to that of invasive species, an established factor of global change ${ }^{1,2}$. The pandemic's origins (as with previous infectious disease outbreaks like Zika virus and SARS) are also intertwined with global change: urbanization, a form of land-use change, and in particular urban encroachment into wildlife habitats, likely contributed to SARS-CoV-2 jumping from a wild animal host to humans, although alternative hypotheses are still being evaluated. The impact of the pandemic on human activities is having environmental consequences that link to other factors of global change ${ }^{3}$. Short-term lockdowns and longer-term changes to travel and working patterns could affect levels of atmospheric greenhouse gases, in turn potentially causing feedback to climate variables. Fighting the pandemic has led to increased production of single-use plastics in the form of medical waste, protective wear, masks and take-away food packaging ${ }^{4}$, some of which finds its way into the environment. Upon fragmentation, this plastic can turn into microplastic (or nanoplastic), a recently recognized aspect of global change ${ }^{5}$ that can affect terrestrial and aquatic ecosystems. Similarly, increased and widespread use of chemicals for disinfection purposes, including antiviral agents and surfactants, can lead to increased abundances of such chemicals in the

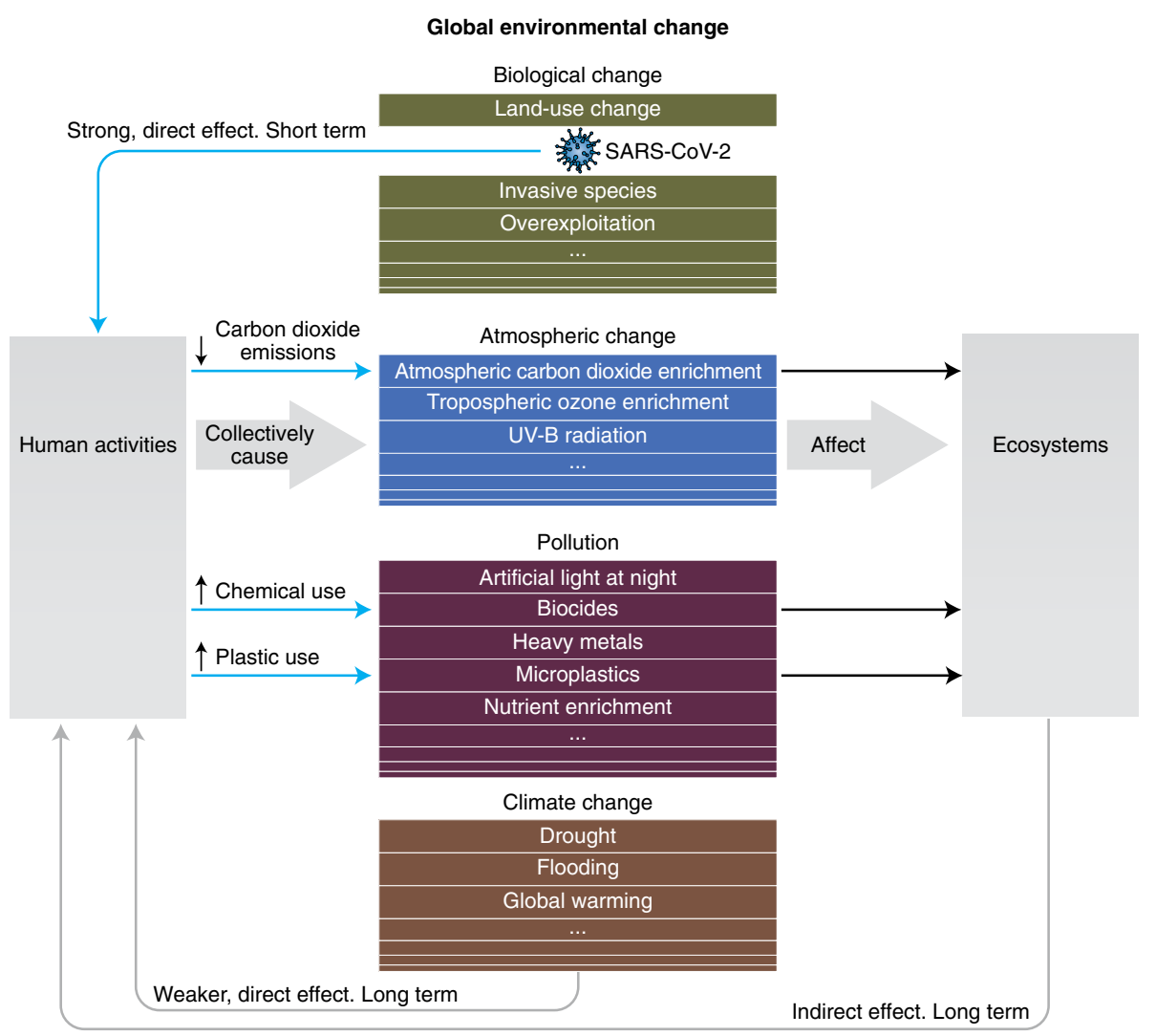

Fig. 1 | The SARS-CoV-2 virus causing the COVID-19 pandemic is linked to a range of global-change factors. Here, we have grouped it under biological change alongside invasive species - the emergence of SARS-CoV-2 as a human pathogen is most likely the result of human-wildlife contact, and its global spread resembles, in some ways, the process of invasive species spread ${ }^{2}$. Global change is ultimately driven by human activities, and affects ecosystems, with feedbacks to human activities mostly long-term and indirect. Since effects of COVID-19 on human activities are strong and immediate, several other global change factors are impacted by the pandemic (blue arrows).

environment, contributing not only to environmental pollution but also potentially to proliferation of resistance genes ${ }^{6}$. Climate change is itself linked in multiple ways to emerging infectious disease, global food security and public health ${ }^{7}$, adding another layer of interactions within this complex set of relationships.

There are also similarities in how key aspects of pandemics and global change factors unfold ${ }^{8}$. They are both high-momentum trends, causing mostly irreversible change. They both tend to induce a deterioration in international cooperation, are less costly to prevent than to fix and exacerbate inequality among citizens, countries and regions. Global change and pandemics occur within common spheres and highlight the evolutionarily wired human ability to deal with the here and now, at the cost of dealing effectively with planning for the future ${ }^{9}$. As a consequence, pathways to solutions, even though they will differ greatly in terms of actual measures or research priorities, are similar between pandemics and many issues connected to global change factors. At the heart is international cooperation, 
which is indispensable for reaching globally relevant goals.

We argue that closely aligning the COVID-19 pandemic (and any future ones) with the topic of global change is crucial, for several reasons. The first and perhaps most important reason is addressing the problem of 'limited bandwidth' or, more specifically, the 'finite pool of worry'. It is possible that the media and political focus on the pandemic, as well as immediate impacts on individuals' lives, will move progress on combatting many aspects of global change backwards in the political agenda and in people's minds (although one recent study showed that at least climate change stayed as a priority for citizens despite the pandemic ${ }^{10}$ ). Scientists adopting a narrative emphasizing that the COVID-19 pandemic is intricately intertwined with global change would help in terms of presenting a more unified message to the public, and to policymakers.

We suggest that much could be improved if we adjusted curricula and educational messages to emphasize the inter-relatedness of the pandemic and global environmental change. This could, for example, mean more systematic teaching of high school and university students about pandemics in environmental science and ecology courses, and about global change in the medical or environmental health fields. In academic research, there should be greater numbers of staff positions and funded programmes that explicitly focus on research at these disciplinary interfaces. Scientists and science communicators should use these joint narratives in their messages to the media, and thus inform the public about the multiple connections between pandemics and global change. Inspiration could come from the long-standing 'One Health' concept, the idea that human well-being is best served if human, animal and ecosystem health are considered together as inter-related components; through coining the term One Health, integration across disciplines has been favoured and more effectively communicated.

But the place where more-integrated messaging is most needed is to policymakers. Global cooperation is necessary to address issues of global change, because neither viral variants nor plastic rain stop at borders. For climate change, there is the Intergovernmental Panel on Climate Change (IPCC); the World Health Organization (WHO) deals with global disease outbreaks; for chemical pollutants and waste there are existing legally binding, environmental conventions and recent calls for a global science policy body ${ }^{11}$; and the Intergovernmental Platform on Biodiversity and Ecosystem Services (IPBES) has recently called additionally for an international body dealing specifically with pandemics ${ }^{12}$. Policymakers in collaboration with scientists need to devise an international policy instrument that deals with global change in its entirety: all human impacts on our planet, focusing especially on the cross-links between different topics. This could mean broadening the scope of existing entities, an increased focus on interaction between existing bodies, such as through more regular joint workshops, or an entirely new organization dealing with all facets of global change in an integrated fashion. Whatever route is taken, it is time to more explicitly consider the interdependencies among the different anthropogenically caused global change challenges, not least because there may be common pathways to solutions.

If we understand, communicate and act on global change as the multi-faceted complex of issues that it really is, this could mark a shift towards achieving a more sustainable Anthropocene.
Matthias C. Rillig (D) 1,2凶, Anika Lehmann (D) 1,2, Michael S. Bank ${ }^{3,4}$, Kenneth A. Gould ${ }^{5}$ and Hauke R. Heekeren (D)6

${ }^{1}$ Institute of Biology, Freie Universität Berlin, Berlin, Germany. ${ }^{2}$ Berlin-Brandenburg Institute of Advanced Biodiversity Research, Berlin, Germany. ${ }^{3}$ Institute of Marine Research, Bergen, Norway. ${ }^{4}$ University of Massachusetts, Amherst, MA, USA. ${ }^{5}$ School of Humanities and Social Sciences, Brooklyn College, City University of New York, New York, NY, USA.

${ }^{6}$ Department of Education and Psychology, Freie Universität Berlin, Berlin, Germany.



Published online: 2 September 2021

https://doi.org/10.1038/s41559-021-01552-7

References

1. Sage, R. F. Global Change Biol. 26, 3-30 (2020).

2. Nuñez, M. A., Pauchard, A. \& Ricciardi, A. Trends Ecol. Evol. 35 , $642-645(2020)$

3. Diffenbaugh, N. S. et al. Nat. Rev. Earth Environ. 1, $470-481(2020)$

4. You, S., Sonne, C. \& Ok, Y. S. Science. 368, 1438-1438 (2020).

5. Rillig, M. C. \& Lehmann, A. Science. 368, 1430-1431 (2020).

6. Zhu, Y.-G. et al. Science. 357, 1099-1100 (2017).

7. Watts, N. et al. Lancet. 386, 1861-1914 (2015).

8. Manzanedo, R. D. \& Manning, P. Sci. Total Environ. 742, 140563 (2020).

9. Weber, E. U. Nat. Hum. Behav. 1, 0013 (2017).

10. Evensen, D. et al. Proc. Natl Acad. Sci. USA 118, e2018936118 (2021).

11. Wang, Z. et al. Science. 371, 774-776 (2021).

12. Workshop Report on Biodiversity and Pandemics of the Intergovernmental Platform on Biodiversity and Ecosystem Services (IPBES Secretariat, 2020).

Acknowledgements

M.C.R. acknowledges funding from an ERC Advanced Grant (grant no. 694368).

Author contributions

M.C.R. conceptualized the structure and content of the manuscript and wrote an initial draft. A.L., M.S.B., K.A.G. and H.R.H. expanded upon the ideas contained within the draft and helped edit the final manuscript. A.L. designed the figure.

Competing interests

The authors declare no competing interests. 